\title{
Rare case of intracytoplasmic sperm injection - embryo transfer twin pregnancy in a patient with unicornuate uterus
}

\author{
Tejal Poddar*, Purnima Nadkarni, Aditi Nadkarni
}

Nadkarni Hospital, Killa Pardi, Gujarat, India

Received: 05 June 2016

Accepted: 01 July 2016

\author{
*Correspondence: \\ Dr. Tejal Poddar, \\ E-mail: tejalpoddar@gmail.com
}

Copyright: (c) the author(s), publisher and licensee Medip Academy. This is an open-access article distributed under the terms of the Creative Commons Attribution Non-Commercial License, which permits unrestricted non-commercial use, distribution, and reproduction in any medium, provided the original work is properly cited.

\begin{abstract}
Congenital uterine anomalies result from an abnormal formation, fusion or reabsorption of Müllerian ducts during fetal life. A 26 year-old healthy woman with history of 5 years of primary infertility. Her physical exam was completely normal, while a transvaginal ultrasound revealed a left unicornuate uterus and normal left ovary and fallopian tube, but the right ovary and tube could not be visualized and right sided ectopic kidney at the level of umbilicus was noted. Left kidney was in normal position. A diagnostic laparoscopy for her confirmed left unicorunate uterus with normal left ovary and tube along with rudimentary right horn and only about $2 \mathrm{~cm}$ proximal stump of the right fallopian tube. The couple underwent two cycles of ICSI because of male factor. First $\beta$ HCG report was $349 \mathrm{mIu} / \mathrm{ml}$ followed by second which was $2432.1 \mathrm{mIu} / \mathrm{ml}$. First ultra sound revealed biamniotic bichorionic twin live intrauterine pregnancies. A fetal reduction was planned at 11 weeks for fetus A due to increases NT $>5 \mathrm{~mm}$ which was completed uneventfully. Cervical length was measured at $18-20$ weeks which were merely $1.4 \mathrm{cms}$. Hence McDonald's cervical encirclage was performed. Cervical knot was cut and emergency cesarean section was performed at 35 weeks gestation, and a $2.6 \mathrm{~kg}$ male baby was delivered uneventfully.
\end{abstract}

Keywords: Congenital uterine anomalies, Müllerian ducts, unicornuate uterus, ICSI pregnancy, Embryo reduction, McDonald's encirclage

\section{INTRODUCTION}

Congenital uterine anomalies result from an abnormal formation, fusion or reabsorption of Müllerian ducts during fetal life. These anomalies are present in 1 to $10 \%$ of the unselected population, 2 to $8 \%$ of infertile women and 5 to $30 \%$ of women with a history of miscarriages. ${ }^{1}$ A unicornuate uterus is present in $0.1 \%$ of the unselected population. The reproductive performance of women with unicornuate uterus is poor, with a live birth rate of only $29.2 \%$, prematurity rate of $44 \%$, and an ectopic pregnancy rate of $4 \% .^{2}$ Moreover, women with anomaly, present with rates of $24.3 \%$ first trimester abortion, $9.7 \%$ second trimester abortion and $10.5 \%$ intrauterine fetal demise. $^{3}$ We present a case report of a successful intracytoplasmic sperm injection - embryo transfer (ICSIET) pregnancy in a unicornuate uterus in an infertile women.

\section{CASE REPORT}

A 26 year-old healthy woman with history of 5 years of primary infertility presented to us for evaluation. The patient had no past surgical history or medical history and her BMI was $21.6 \mathrm{~kg} / \mathrm{m}^{2}$. She was treated unsuccessfully for 6 months with clomiphene citrate ( $50 \mathrm{mg} /$ for 5 days) and intrauterine inseminations (IUI) in another center. Routine assessment of her ovarian reserve was in the upper range of normal (cycle day 3 FSH: 8.8 mIU $\backslash \mathrm{mL}$; E2:75; LH: $9.0 \mathrm{mIU} \backslash \mathrm{mL}$ and Anti-Mullerian Hormone (AMH) (3.48ng $\backslash 100 \mathrm{~mL})$ PRL was normal (22 $\mathrm{ng} \backslash \mathrm{mL})$ and TSH was normal (1.82 mIULL). Her karyotype was normal (46 XX). The Pap smear was normal. Her physical exam was completely normal, HSG revealed a left unicotnuate uterus (Figure 1), while a transvaginal ultrasound revealed a left unicornuate uterus and normal left ovary and fallopian tube, but the right ovary and tube 
could not be visualized and right sided ectopic kidney at the level of umbilicus was noted. Left kidney was in normal position. A diagnostic laparoscopy for her confirmed left unicorunate uterus with normal left ovary and tube along with rudimentary right horn and revealed only about $2 \mathrm{~cm}$ proximal stump of the right fallopian tube.

Chromopertubation using diluted methylene blue dye showed fill and spill of the left fallopian tube while on the right there was proximal fill of the short cornual portion, but no spill. The couple underwent two cycles of ICSI because of male factor (sperm concentrations: 5 mil $\backslash \mathrm{ml}$; motility $30 \%$; normal morphology: 45\%). The ovarian stimulation for the first ICSI cycle consisted of recombinant FSH (rFSH) (with a total rFSH dose of 1912.5 IU) and GnRH antagonist; the maximum E2 level was $2180 ; 10$ oocytes were recovered but only 4 fertilized; 2 embryos were transferred on day 3 but no pregnancy resulted. For the second ICSI cycle the same protocol, except for higher doses of $\mathrm{rFSH}$, was used (total rFSH 3000 IU and the maximum level E2 was 2980; 12 oocytes were retrieved, eight fertilized and two embryos were transferred on day 5. First $\beta$ HCG report was $349 \mathrm{mIu} / \mathrm{ml}$ followed by second which was $2432.1 \mathrm{mIu} / \mathrm{ml}$. First ultra sound revealed biamniotic bichorionic twin live intrauterine pregnancies. Sequential scan done at 10 weeks gestation revealed one sac having oligohydramnios along with cystic hygroma with increased NT measuring $>5 \mathrm{~mm}$ of fetus A (Figure 2). Fetus B revealed normal ultrasound scan corresponding to weeks of amenorrhea. A fetal reduction was planned at 11 weeks for fetus A which was completed uneventfully with no complications. She was followed up throughout with regular antenatal check-up visits. She was given progesterone support from embryo transfer till 34 weeks. Progesterone support included vaginal progesterone, 400 mg twice daily.

Cervical length was measured at $18-20$ weeks which were merely $1.4 \mathrm{cms}$. Hence McDonald's cervical encirclage was performed. Pregnancy carried uneventfully till 35 weeks gestation at which she developed uterine contractions and she went into labour. Cervical knot was cut and emergency cesarean section was performed, and a $2.6 \mathrm{~kg}$ male baby was delivered uneventfully (Figure 3).

\section{DISCUSSION}

A unicornuate uterus is a type II classification with unilateral hypoplasia or agenesis that can be further sub classified into communicating, noncommunicating, no cavity and no horn. ${ }^{4}$ A rudimentary horn with unicornuate uterus results from failure of complete development of one of the Müllerian ducts associated with the incomplete fusion of the contralateral one.

Nanda et al described a successful twin pregnancy in a unicornuate uterus with one fetus in the non- communicating rudimentary horn, but many other cases of ruptured non-communicating rudimentary horn pregnancies have been described. ${ }^{6,7}$

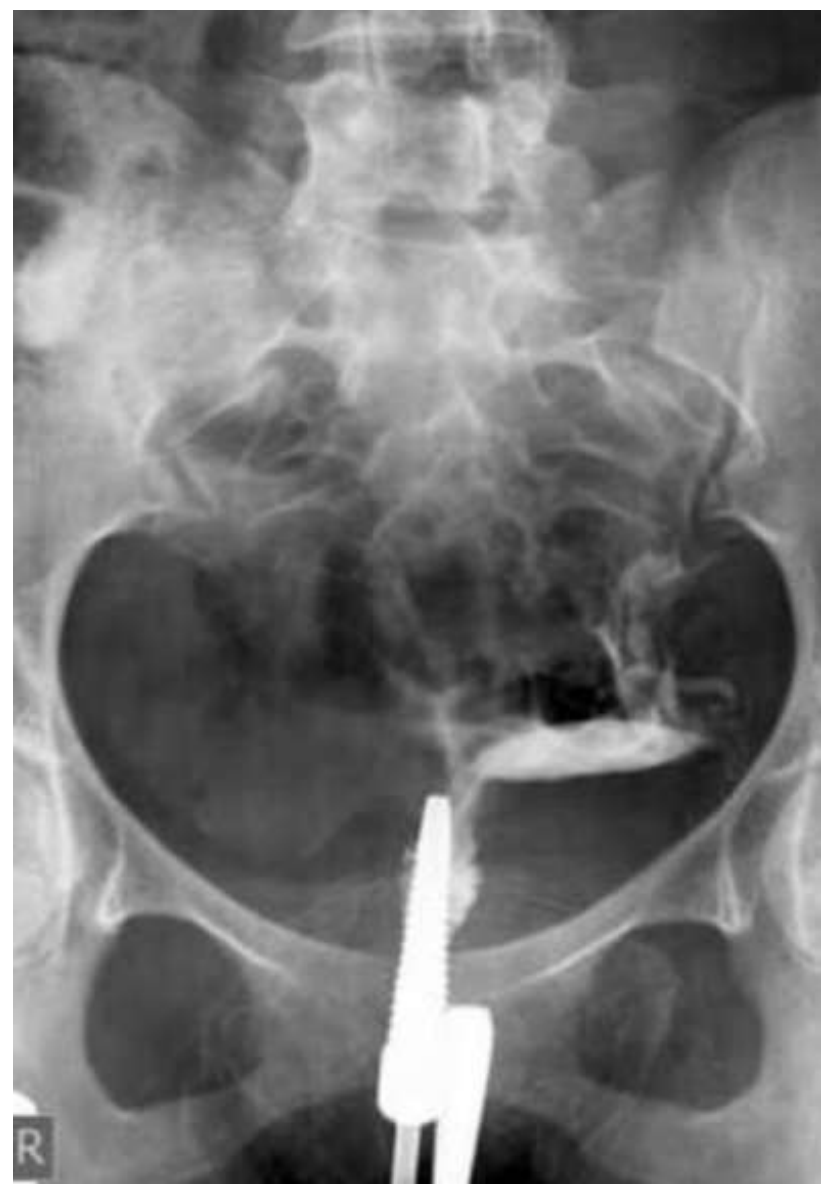

Figure 1: HSG revealed a left unicotnuate uterus.

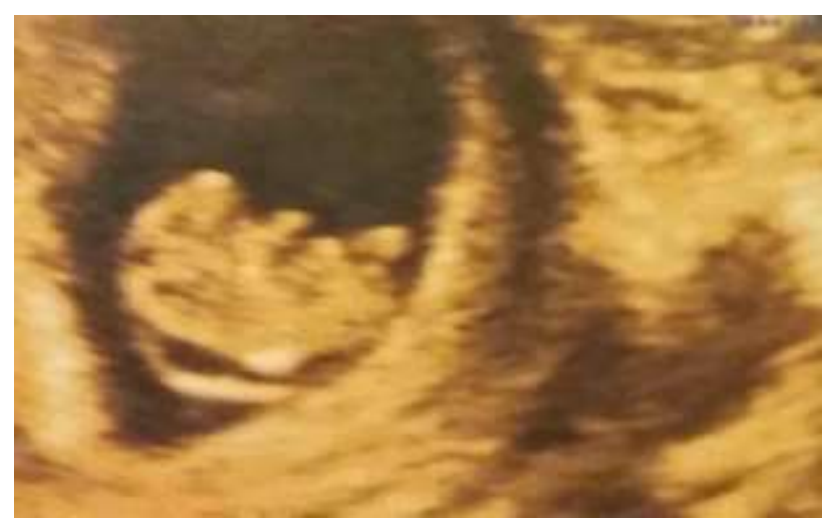

Figure 2: Sequential scan at 10 weeks gestation revealed one sac having oligohydramnios along with cystic hygroma.

Literature data shows that, in the general population, losses occur due to uterine anomalies with a frequency of approximately $15 \% .^{8}$ In the group of patients with recurrent miscarriages, malformations of the uterus are diagnosed more often than in the general population $(25 \%$ vs. $7-8 \%){ }^{9,10}$ Zlopasa et al showed in their study a 
significantly higher miscarriage rate in patients with defects of the uterus than in the control group of healthy women $(33.7 \%$ vs. $19.5 \%)$

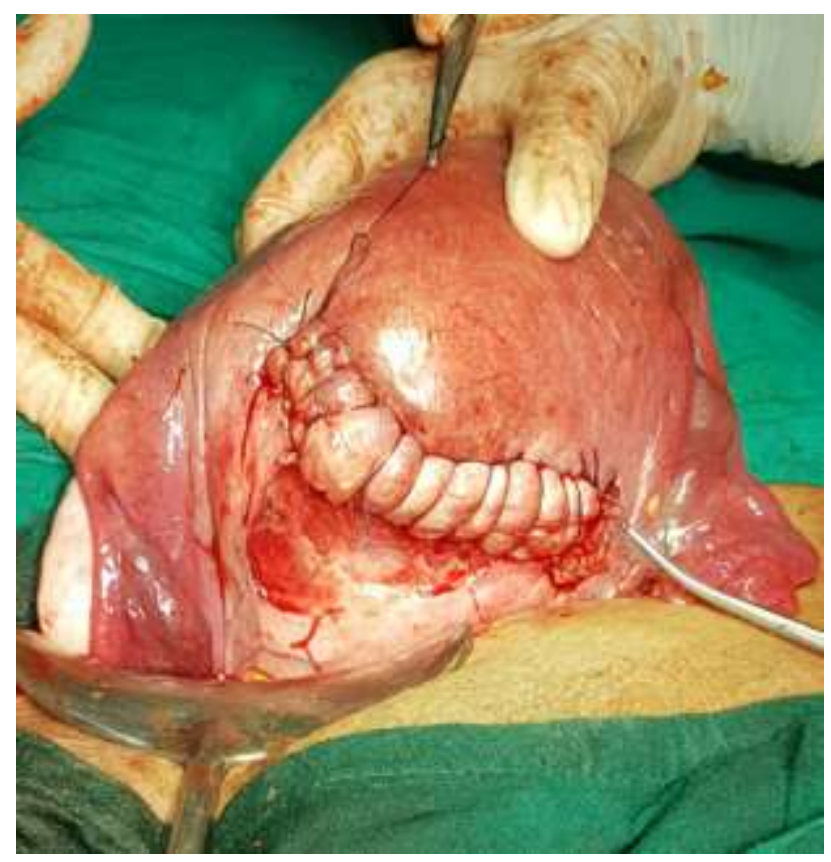

Figure 3: Emergency cesarean section.

The highest percentage of abortions occurred in the group of patients with uterine septum. Zlopasa et al observed significantly worse birth rates in infants of mothers with uterine defects vs. the children from women without such a condition (29\% vs. $9 \%) .{ }^{10}$ Martinez et al conducted an analysis of risk factors for birth defects in children born from mothers with uterine defects, mainly two--horned uterus. They demonstrated in their study that birth of a child with birth $\operatorname{defect}(\mathrm{s})$ is 4 times higher among mothers with uterine defects than in women without such defect. ${ }^{11}$ These defects are due to reduced uterine volumes, where a developing fetus does not have enough space for proper growth, while mechanical pressure impacts the growing fetal structures

Literature provides numerous data on urinary tract defects in women with congenital uterine malformations. ${ }^{12,13}$ Urinary tract defects may affect up to $20-30 \%$ of patients with defects of the uterus, which is probably related to the embryonic development of both systems. Urological counselling is advised in all women with congenital uterine defects

The utility of ultrasound cervix length measurement for assessing the risk of preterm birth has been well documented, with an accepted cut-off value for cervix length of $\leq 25 \mathrm{~mm}$ before the 24 th week of gestational age. The predictive value of a negative test is high (92\%); this implies that pregnant women who do not have a shortened cervix can be reassured, and unnecessary therapeutic measures can be avoided. By contrast, cervical cerclage is the best treatment for women with a short cervix $(<25 \mathrm{~mm})$, and particularly for women with a history of prior midtrimester pregnancy losses due to cervical insufficiency, Therefore, in our case report, a cervical cerclage was considered necessary.

Recent data suggest that progesterone may be important in maintaining uterine quiescence in the latter half of pregnancy by limiting the production of stimulatory prostaglandins and inhibiting the expression of contraction associated protein genes (ion channels, oxytocin and prostaglandin receptors, and gap junctions) within the myometrium. The role of progesterone in later pregnancy, however, is less clear. In fact, ACOG recommend progesterone supplementation only for prior spontaneous preterm birth and cervical shortening ( $\leq 15 \mathrm{~mm}$ prior to 24 weeks) so we decided to administer this treatment in our present case. Although a premature birth can also be due to premature contractions, a tocolytic therapy is suggested in this situation. In our case report it was considered useful to perform serial growth ultrasound examinations for assessing a possible IUGR and an ultrasound cervix length measurement to assess the risk of preterm birth, and to prescribe a ritodrine tocolytic therapy when contractions led to shortening of the cervix length. Our case report shows that by adopting these strategies the prognosis of pregnancy in a unicornuate uterus is not always impaired, although breech presentation, cesarean delivery and prematurity threatens to occur.

Nevertheless, the optimal management approach cannot be clearly stated. Further large observational and prospective studies are essential to investigate the treatments needed during pregnancies in this uterine anomaly.

\section{CONCLUSION}

The true population prevalence of congenital uterine anomalies is difficult to assess partly because there are no universally standardized classification systems and partly because the best diagnostic techniques are invasive, therefore, they are rarely applied to low-risk study populations. Reproductive outcome of IVF-ET in a uterine anomaly in women is different from women with normal uterine cavity. Multiple pregnancies may have a negative impact on the likelihood of a full term pregnancy; therefore, every effort should be made to achieve a singleton pregnancy in these patients.

Pregnancy in a woman with uterine defects should be regarded as a high-risk condition. Intensive monitoring of such pregnancy and of delivery, as well as a package of preventive measures to avoid possible complications, is highly indicated.

\section{Funding: No funding sources Conflict of interest: None declared Ethical approval: Not required}




\section{REFERENCES}

1. Chan YY, Jayaprakasan K, Tan A, Thornton JG, Coomarasamy A, Raine- Fenning NJ. Reproductive outcomes in women with congenital uterine anomalies: a systematic review. Ultrasound Obstet Gynecol. 2011;38:371-82.

2. Akar ME, Bayar D, Yildiz S, Ozel M, Yilmaz Z. Reproductive outcome of women with unicornuate uterus. Aust N Z Obstet Gynaecol. 2005;45(2):14850.

3. Reichman D, Laufer MR, Robinson BK. Pregnancy outcomes in unicornuate uteri: a review. Fertil Steril 2009;91(5):1886-94.

4. Gordts S, Brucker S, Gergolet M, Tanos V, Li T-C, De Angelis C, Di Spiezio Sardo A. Clinical approach for the classification of congenital uterine malformations. Gynecol Surg. 2012;9:119-29.

5. Nanda S, Dahiya K, Sharma N, Aggarwal D, Sighal SR, Sangwan N. Successful twin pregnancy in a unicornuate uterus with one fetus in the noncommunicating rudimentary horn. Arch Gynecol Obstet. 2009;280(6):993-5.

6. Amritha B, Sumangali T, Priya B, Deepak S, Sharadha R. A rare case of term viable secondary abdominal pregnancy following rupture of rudimentary horn: a case report. J Med Case Rep. 2009;3:38.
7. Kanagal DV, Hanumanalu LC. Ruptured rudimentary horn pregnancy at 25 weeks with previous vaginal delivery: a case report. Case Rep Obstet Gynecol. 2012:985076.

8. Bhattacharya S, Bhattacharya S. Effect of miscarriage on future pregnancies. Women's Health. 2009;5:5-8

9. Acien P. Reproductive performance of women with uterine malformations. Hum Reprod. 1993;8:122-6.

10. Zlopasa G, Skrablin S, Kalafatic D, Banović V, Lesin J. Uterine anomalies and pregnancy outcome following resectoscope metroplasty. Int J Gynecol Obstet. 2007;98:129-33.

11. Martinez-Frias ML, Bermejo E, Rodriguez-Pinilla E, Frías JL. Congenital anomalies in the offspring of mothers with a bicornuate uterus. Pediatrics. 1998;101:693-4.

12. Raga F, Bauset C, Remohi J, Bonilla-Musoles F, Simón C, Pellicer A. Reproductive impact of congenital mullerian anomalies. Hum Reprod. 1997;12:2277-81.

13. Jayasinghe Y, Rane A, Stalewski H, Grover S. The presentation and early diagnosis of the rudimentary uterine horn. Obstet Gynecol. 2005;105:1456-67.

Cite this article as: Poddar T, Nadkarni P, Nadkarni A. Rare case of intracytoplasmic sperm injection embryo transfer twin pregnancy in a patient with unicornuate uterus. Int J Reprod Contracept Obstet Gynecol 2016;5:2862-5. 